L.A. Willson

Astronomy Program, Physics Department

Iowa State University, Ames IA 50011, USA

Abstract. Mass loss at rates sufficient to alter the evolution of stars is known to occur during the pre-main sequence evolution of most stars, on the main sequence for massive stars, and during advanced evolutionary phases when the luminosity is high and the effective temperature is low. While most investigations of the effects of mass loss on stellar evolution have assumed continuous (parametrized) mass loss laws apply, there is increasing evidence that mass loss rates are substantially higher for stars that are pulsating with large amplitude and/or in selected modes. Some new insights into the mass loss that terminates the AGB evolution of intermediate mass stars, and leads to the formation of planetary nebulae, come from recent detailed studies of the mass loss process from the Mira variables.

\title{
INTRODUCTORY COMMENTS
}

Any systematic study of mass loss from stars must include three parts: detailed calculations of the mechanisms that drive the mass loss; observations of the mass flux, temperature structure and velocity structure of the winds; and the inclusion of the mass loss in stellar evolution calculations. Current studies mostly fall short of these ideal goals; only for the case of the late asymptotic giant branch (AGB) Mira variables are at least some results available in all three categories.

Mass loss: general considerations

To lift material from the surface of a star and drive it away as a wind requires the input of both energy $\left(L_{\dot{M}} \approx 0.5 \dot{M}\left(v_{e}^{2}+v_{\infty}^{2}\right)\right)$ and momentum flux $\left(\approx \dot{M} v_{\infty}\right)$. The condition $L_{M}<L_{*}$ gives

$$
\dot{\mathrm{M}} \leqq 4.9 \times 10^{-8} \operatorname{LR} \mathrm{M}^{-1}\left(1+\mathrm{v}_{\infty}^{2} / \mathrm{v}_{e}^{2}\right)^{-1}
$$

where $L, R$, and $M$ are in solar units. For the case where the momentum is provided by radiative driving, the momentum condition

$$
\dot{M} v_{\infty}=(4 \pi / c) \quad \int_{r_{0}}^{\infty} r^{2} d r \int_{0}^{\infty} d \nu X_{\nu} F_{\nu}
$$

also applies, with $X_{\nu}=$ total extinction coefficient (Mihalas 1978, \$2-3). For an optically thin envelope, $F_{\nu}=F_{\nu}$ (star) $+F_{\nu}$ (cs envelope) $\approx \mathrm{F}_{\nu}\left(\right.$ star), and Equation 2 reduces to $\dot{M}_{\infty}=\langle\tau\rangle_{F} L_{*} / \mathrm{c}$ (with 
$\langle\tau\rangle_{F}=$ flux-averaged optical depth). Knapp (1986; Knapp et al. 1982) defined a factor $\beta=L /\left(M_{C} v_{\infty}\right)$ that is $<1$ for most radiatively driven stellar winds. It is possible to get $\beta>1$ if, for example, there is an opaque circumstellar shell with a transparent cavity near the star, allowing photons to scatter several times across the cavity, so that a dust particle in the inner shell sees $F_{\nu}$ (cs envelope) $\approx F_{\nu}$ (star). Such a configuration arises naturally for an OH-IR source with a moderately warm central star; then the dust forms at some distance above the stellar photosphere, and the opacity increases abruptly where the dust forms.

Virtually all studies of the effects of mass loss on stellar evolution have assumed that there is a continuous parametrizable mass loss relation that applies to nearly all stars, such as the relation (Reimers 1975):

$$
\dot{M}=\eta_{R} 4 \times 10^{-13} \mathrm{LR} \mathrm{M} \mathrm{M}^{-1} \mathrm{M}_{\odot} \cdot \mathrm{Yr}^{-1} \text {. }
$$

This is the same as equation (1) if $\eta_{R}=8 \times 10^{4} a\left(1+v_{\infty}^{2} / v_{e}^{2}\right)^{-1}$, where $\mathbf{a}$ is a measure of the efficiency of the wind (if $v_{\infty} \ll<v_{e}, \quad a=L_{M} / L_{*}$ ) $\dot{m}_{-6}$ Observed mass loss rates correspond to a wide range of $\boldsymbol{\alpha}$, from $\boldsymbol{\alpha} \leqq 10^{-6}$ for the sun to $>10^{-2}$ for some pre-main sequence stars.

Reimers originally obtained equation (3) with $\eta_{R}=1$ from a fit to observed mass loss rates for red giants. Subsequent testing of this relation in evolutionary models indicated that this overestimated the average mass loss rate, and currently favored representative values of $\boldsymbol{\eta}_{\mathrm{R}}$ are $\sim 1 / 4$ to $1 / 3$ (see e.g. Reimers 1987). Note that these values are sufficiently low as to imply very little mass loss over most of the evolution of intermediate and low mass stars. For example, for $\eta_{R}=.25$, at most $\sim 0.01 \mathrm{M}_{\odot}$ is expected to be lost on the first giant branch (RGB), while the existence of blue horizontal branch stars and RR Lyrae stars requires that at least $0.1 \mathrm{M}_{0}$ be lost from stars on the RGB (Rood 1973). It has become quite clear that there is no single value of $\eta_{R}$ that can satisfy observational constraints, but rather, that there are "episodes" of enhanced mass loss that are most important in the evolutionary picture.

Pulsation and mass loss

Hydrodynamical models for the atmospheres of pulsating stars have been calculated by Hill (Hill \& Willson 1979, Willson \& Hill 1979 collectively $=\mathrm{HW}$ ), by Wood (1979), and by Bowen (1988) for the Mira variables; an analytic understanding of these calculations is described in some detail by HW and by Willson \& Bowen (1985, 1986a, 1986b collectively $=\mathrm{WB}$ ). A few explorations of the effects of pulsation on the atmospheres of other categories of variable stars have been made by Hill (1972, 1975) for RR Lyrae stars and $\partial$ Scuti stars, and by Bowen (unpublished) for Cepheids and RR Lyrae stars; but these so far lack essential details of the thermal and radiative physics, so only adumbrate the results of more detailed calculations to come. 
Essential results from the model calculations, as understood by the analytic theory, reveal that pulsation quite generally leads to an increased scale height in the stellar atmosphere, lifting substantial amounts of material to an altitude where other mechanisms can act more effectively to drive it away. Pulsation is capable of greatly increasing the density in the outer atmosphere even for stars with small static scale heights. The increase in the density at a given altitude for a given set of stellar parameters depends on the pulsation mode and on the driving amplitude (or, more physically, on the fraction of the pulsation damping that is contributed by the atmosphere). The increase in the scale height in the region containing atmospheric shocks depends mainly on the pulsation mode. The density at which the shocks form depends also on the driving amplitude: stronger driving in a given mode $\rightarrow$ deeper shocks $\rightarrow$ higher densities in the outer atmosphere.

Pulsation alone can drive mass loss (for example by also heating the atmosphere to drive a thermal wind) but in most cases where substantial mass loss occurs there is also an important second mechanism involved. Examples of second mechanisms that have been at least tentatively identified include radiative forces ("radiation pressure") on dust in Miras, rapid rotation in Be stars and main sequence $A$ stars, radiative forces due to the high opacity in UV resonance lines in $O$ and $B$ stars, and the absorption of Lyman line and continuum emission (originating from shocks or in an extended calorisphere; WB) in Cepheids (Willson \& Bowen $1984=$ WB*$^{*}$ Brunish \& Willson 1987 and in preparation).

\section{IMPORTANT MASS LOSS STAGES IN STELLAR EVOLUTION}

There are only a few classes of stars for which there is clear, unambiguous evidence that important mass loss is occurring: premain sequence objects, luminous early-type stars, and highly evolved red giants or supergiants. Winds have also been observed coming from $R \mathrm{Cr} B$ stars, planetary nebula nuclei, Be stars and Wolf-Rayet stars, but it is not yet clear whether these represent mass loss sufficient to affect the (already rapid) evolution of these objects. Winds have been proposed to occur (as the result of pulsation) for Cepheids, RR Lyrae stars, and main sequence A stars (WB*; Willson, Bowen \& Struck-Marcell $1987=W B S$ ) but these winds have yet to be confirmed by direct measurement.

\section{Pre-main sequence mass loss}

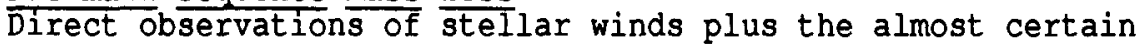
necessity for protostars to shed angular momentum during their evolution to the main sequence provide persuasive evidence that stars lose substantial mass before reaching the main sequence. As yet there has been no detailed investigation of the possibility that pulsation plays a role in this process, although the Hayashi tracks for stars with $M<1.5 M_{0}$ lie within the region of Mira instability and pulsation is known to drive substantial mass loss from the (post-main sequence) Miras. There are two complications facing investigations concerning the possible role of pulsation in pre-main sequence evolution and mass loss (Willson 1988): (1) the stars are not directly observable until they 
leave the Mira region (Stahler's (1983) "birth line" lies on or near the blue edge of the Mira strip as determined from post-main sequence Miras), complicating observations, and (2) rotation is likely to play an important role both by affecting the pulsation mode(s) and in enhancing or modulating the mass loss, greatly complicating the theory.

\section{Main sequence mass loss}

On the upper main sequence, $O$ and early $B$ stars $\left(M>\sim 5 M_{\odot}\right)$ have observed mass loss rates high enough to affect the evolution of at least the more massive $\left(>10 \mathrm{M}_{\odot}\right)$ stars (Chiosi \& and Maeder 1986). The mechanism in this case is the transfer of momentum from the radiation field to the gas via resonance line absorption by abundant ions (Castor, Abbott \& Klein 1975). The rapidly rotating Be stars also show substantial if episodic mass loss, and the possibility that this is closely linked to the pulsation properties has been considered (Smith 1988; Smith \& Penrod 1985; Willson 1986).

Stars between 1+ and 2+ solar masses (early A to mid F spectral types) lie on the main sequence in the region of the "Cepheid" instability strip. Some of these are observed to pulsate as $\delta$ Scuti stars but most have small amplitudes or no apparent variability, even though theoretical calculations indicate that these should be quite pulsationally unstable (Stellingwerf 1979). Stellingwerf noted that one explanation for the lack of obvious pulsation is that there is atmospheric damping associated with the driving of substantial winds from these stars. WBS suggested that mass loss associated with pulsation combined with rapid rotation in this part of the main sequence could cause stars to evolve down the main sequence. Such evolution would invalidate the standard method of dating clusters that are older than about a billion years, possibly providing a solution to the problem that standard dating of globular clusters yields ages of 14-18 Gyr while other methods of dating the galaxy and the universe appear to favor ages $<12$ Gyr (WBS). This hypothesis would also provide a novel explanation for the blue stragglers as being those stars that (due to slow rotation or lying to the blue of the instability strip) did not share in the general downward migration of the rapidly rotating, pulsating and hence mass-losing stars. It would predict (or account for) the observed deficiency of $A$ and early $F$ stars (that indicates a deficiency of stars with masses between about 1.2 and 2 to $3 \mathrm{M}_{\odot}$ if standard mass-spectral type calibrations are used). The level of mass loss needed to produce evolution down the main sequence is sufficiently low that direct detection may be difficult, depending on the temperature of the outflowing gas. Many A stars have been found to have infrared excesses in the IRAS survey, though these may be accounted for by the presence of a remnant proto-planetary disk of relatively large solid grains (Aumann et al. 1984; Gillett 1986).

Post-main sequence mass loss

For evolved low mass, Population II, stars there is evidence for mass loss near the tip of the red giant branch; without such mass 
loss, there would be no RR Lyrae stars (Rood 1973). This mass loss may be associated with a brief stage of Mira-like pulsations. Mass loss associated with RR Lyrae pulsation may also play a significant role in populating the blue horizontal branch $\left(\mathrm{WB}^{\star}\right)$, causing the blueward evolution of RR Lyrae stars to occur at higher luminosities than expected, and concentrating the RR Lyrae stars along the blue edge and fundamental blue edge of the instability strip. RV Tauri stars show Mira-like, somewhat irregular, pulsations; many RV Tauri stars also show IR excesses indicating substantial current or recent past mass loss (Gehrz \& Woolf 1970; Raveendran \& Rao 1988).

\section{Do Cepheids lose mass?}

Stars with initial masses between 3 to $5 \mathrm{M}_{\odot}$ and about $15 \mathrm{M}_{\odot}$ pass through the Cepheid instability strip during their core-He burning blueward loops (Becker 1981); the possibility that significant mass loss occurs as a result of Cepheid pulsation for at least the shorter period, lower mass Cepheids was suggested by WB*. It is not yet clear whether such mass loss affects the evolution of the Cepheids: Theoretical models confirm that it is possible to "trap" the Cepheids in the instability strip with mass loss, to reduce the masses substantially and thus possibly solve the "Cepheid Mass Anomaly" (Cox 1980 - but see also Andreason 1988), and that this reduces the rate of change of the periods to better agreement with those observed (Brunish \& Willson 1987 and in preparation). However, the critical mass loss rate required to produce these effects is relatively high (from $10^{-7} \mathrm{M}_{\circ} \cdot \mathrm{yr}^{-1}$ for a $5 \mathrm{M}_{0}, 3-5$ day model to $10^{-5} \mathrm{M}_{\odot} \cdot \mathrm{yr}^{-1}$ for an $8 \mathrm{M}_{0}, 10$ day model). Observations in the radio and infrared spectral regions seem to exclude such high rates of flow either as a fully ionized plasma or as a cool, dusty wind (Butler 1987, MacAlary \& Welch 1986, Welch \& Duric 1988). There is some indication of a blueshifted, steady line component at about 30-50 $\mathrm{km} \cdot \mathrm{sec}^{-1}$ in the UV spectra (Schmidt \& Parsons 1984), consistent with an outflow in which the absorption of line radiation (La, particularly) from deep-lying shocks plays a role in driving the flow; this is consistent with a wind that is neither ionized nor dusty. More detailed modeling of the Cepheid atmospheres and winds is needed in order to (1) establish the expected rate of mass loss and (2) allow for the derivation of reliable mass loss rates or limits from the spectral line features.

For stars with initial masses less than about $5 \mathrm{M}_{\odot}$, stars that ultimately become white dwarfs with $M \leqslant 0.7 M_{\odot}$, the most important post-main sequence mass loss occurs near the tip of the AGB. This mass loss stage is closely associated with Mira pulsation, and will be discussed in detail in the next section.

A SYSTEMATIC COMPARISON OF THEORY WITH OBSERVATIONS: MIIRAS AND MASS LOSS ON THE ASYMPTOTIC GIANT BRANCH It is appropriate to devote the largest portion of this review to the Miras and the end of the AGB for two reasons: (1) this is the best-studied and most obvious case of pulsation-related mass loss 
and (2) the mass $103 \mathrm{~s}$ at this stage has important consequences for fundamental problems of astronomy. AGB stars are believed to be the primary source of the grains or the seed-nuclei for interstellar grains, that in turn play an essential role in star formation. Carbon stars are very common and very luminous, and thus may provide a very important "standard candle" for extragalactic distance determinations (Richer 1988). AGB processes determine the mass functions for white dwarfs and for planetary nebulae. An understanding of the processes that terminate the AGB evolution of low/intermediate mass stars is essential for models of nucleosynthesis and galactic chemical evolution. AGB stars are the prime source for s-processed material; stellar mass loss during and following the AGB phase returns this material to the interstellar medium. Also, supernovae provide the $r$-process elements, and the AGB mass loss process probably determines the minimum mass progenitor for supernovae; only when the mechanism is well understood can we expect to determine how this limiting mass varies with metallicity or initial helium abundance.

Properties of individual Miras

The properties of individual Miras should ultimately be determined by matching observed stellar characteristics and spectra to a model that includes both the dynamical effects and the essential radiative transfer to predict radiative fluxes, line profiles, angular diameters and light curves for Miras. Efforts to achieve this synthesis have been undertaken by several investigators, and preliminary results are promising. Beach, Willson \& Bowen $(1988=\mathrm{BWB})$ used dynamical models by Bowen to investigate the effects of the extended atmosphere on angular diameter measurements. Bessell (1989) has calculated spectral energy distributions from simplified dynamical models by Wood, and finds good agreement with the observations.

Comparisons of the dynamical models with the observed velocity variations in the Miras have provided essential evidence that the Miras almost certainly are fundamental mode pulsators. For a given (observed) period distribution, fundamental mode models have smaller radii and hence higher gravities. Shock amplitudes as large as those observed occur only in fundamental mode models (Bowen 1988; see also WB and HW). The greatly extended atmospheres of the Miras, resulting from the combined effects of pulsation and radiative forces, lead to apparent angular diameters that may exceed the "theoretical radius" (where Trosseland $\sim 2 / 3$ ) by factors of 2 or more (BWB); this misled early investigators into believing that the stars were overtone pulsators based on "observed" $Q=P \sqrt{p}$ values.

A study of the MgII emission from Mira variables, carried out with the International Ultraviolet Explorer (IUE) satellite (Willson 1988; Brugel et al. 1986, 1987), provides further confirmation that the nearby Miras are fundamental mode pulsators. The decay of the MgII emission agrees well with the predictions for fundamental mode models (as interpreted, so far, with simplified emission and radiative transfer treatments). According to this interpretation, the MgII emission that is seen is 
generated by the shock as it passes between 1.5 or 2 stellar radii and 3 to 5 stellar radii; thus the MgII emission is a potentially valuable probe of the conditions in the dust-forming part of the stellar atmosphere that the Bowen models suggest plays a critical role in the mass loss process.

Systematic study of AGB evolution with mass loss

By comparing the (parametrized) results of evolutionary and atmospheric models with observed (mostly statistical) properties of the Miras we can determine which mass loss processes are most important as a function of metallicity and progenitor mass. Preliminary results of such a study are presented here; a more detailed report is under preparation by Willson, Kowalsky, Kirpes and Preston.

Required for such a systematic study are: evolutionary tracks (L/M, T, $\mathrm{Z},(\mathrm{H}))$; evolution rate $\left(\mathrm{d}(\log \mathrm{L}) / \mathrm{dt}\right.$ or $\mathrm{L}$ vs. $\left.\mathrm{M}_{\text {Core }}\right)$; pulsation relations ( $\mathrm{P}_{0}$ and $\left.\mathrm{P}_{1}\{\mathrm{M}, \mathrm{R}, \mathrm{Z}, \mathrm{I} / \mathrm{H}\}\right)$; the locus of Mira onset ( $\mathrm{L}_{\text {on }}\{\mathrm{M}, \mathrm{L}, \mathrm{T}, \mathrm{Z}$, $1 / \mathrm{H}\})$; a mass loss function (M $\{\mathrm{L}, \mathrm{M}, \mathrm{R}, \mathrm{Z}$, mode, $1 / \mathrm{H}\}) ;$ and an initial AGB mass function (the I(AGB)MF). The most uncertain relations are the onset locus ( $\mathrm{L}$ vs $\mathrm{T}$ and/or $\mathrm{M}, \mathrm{Z}$ ) of Mira pulsation, the mass loss function, and the I(AGB)MF. The only unobservable (and artificial) parameter is the ratio of convective mixing length to scale height, $1 / \mathrm{H}$.

Constraints on the evolution of AGB stars in L and M from observations include several that are observationally well determined: the periodluminosity relation, the period histogram, the relation between the limiting wind flow speed and the period or luminosity, the initial/final mass relation and the mass functions of white dwarfs and of the central stars of planetary nebulae. Also relatively well determined observationally (although tricky to interpret) are the relative fraction of $M, S$ and $C$ stars as a function of $P, L$ and/or $T$, and the distribution with period of stars with surface Technetium (indicating recent dredge-up of s-processed material; Little-Marenin 1989). Less well determined observationally, but desirable from the theoretical standpoint, are the relations between progenitor mass and period, and the effective temperatures vs. period or luminosity. The shock velocity amplitudes are useful at the level of deciding the mode of pulsation; present data do not provide closer constraints because the geometrical correction factor $v_{r} / v_{\text {obs }}$ (to correct for integration of the line profile over the stellar disk) is quite uncertain.

Mira parameters that are consistent with the period-luminosity relation, some choice of published effective temperatures, and the initial/final mass relation can be chosen either for fundamental or for overtone pulsation (Willson \& Kowalsky 1986). However, as we have indicated, atmospheric models computed for the overtone case fail to reproduce the observed shock amplitudes. In the discussion that follows only the fundamental mode case will be considered. 
Before the observed properties of Mira variables can be compared with the predictions, it is essential to sort the true Miras (fundamental mode AGB stars) from their close relatives (overtone pulsators and/or first giant branch variables). Again, the dynamical atmosphere models and the analytic theory give us guidance. Shock amplitudes in excess of 20-30 $\mathrm{km} \cdot \mathrm{sec}^{-1}$ occur only in fundamental mode pulsators; it is these large shocks that have allowed the identification of the mode of the Miras (WB, HW). Only for a few of the nearest stars have detailed high-resolution near-IR spectra allowed us resolve the velocities of the deep shock. However, for overtone pulsation, regardless of the driving amplitude, the shock amplitude will be too small to produce a lot of hydrogen Balmer emission. For fundamental mode pulsation, all but the lowest amplitude or lowest gravity cases are expected to show $\mathrm{H}$ Balmer emission. Thus the presence of $\mathrm{H}$ Balmer emission lines over a portion of the pulsation cycle is probably the most useful criterion for selecting $F$ mode pulsators. Luminosity provides a criterion for selecting AGB stars, since most lie above the maximum luminosity of the first giant branch. The presence of Technetium lines in the stellar spectrum confirms that we are dealing with a thermally-pulsing AGB star; but some TP-AGB stars may not show Technetium.

Evolutionary models for AGB stars have been recently reviewed by Iben \& Renzini (1983). $\overline{\mathrm{AG} B}$ stars alternate between $\mathrm{H}$ shell burning and He shell flashes, with the pulse length and the duration of the interpulse phase a function of the stellar properties. A troubling source of uncertainty in the modeling is the parametrized mixing length theory of convection; the evolutionary tracks shift to cooler temperatures as the mixing length/scale height ratio is decreased. Unfortunately observations only weakly constrain $T_{\text {eff }}$ as well for the stars near the tip of the $A G B$, the Miras, due to the complications introduced by their highly extended atmospheres (BWB).

A parametrized fit to evolutionary tracks for AGB stars is given by (Iben 1984):

$$
R=312\left(\mathrm{~L} / 10^{4}\right)^{0.68}(1.175 / \mathrm{M})^{0.31 S}(\mathrm{Z} / 0.001)^{0.088}(\mathrm{I} / \mathrm{H})^{-0.52}
$$

where $L, R$, and $M$ are in solar units; $S=1$ for $M>1.175,0$ for $M<1.175$; and $(\mathbf{l} / \mathrm{H})$ is the ratio of the mixing length to the pressure scale height.

Pulsation calculations for Miras have been computed by Ostlie (1982; Ostlie \& Cox 1986) and by Wood (1979; Fox \& Wood 1982, 1985). For fundamental mode pulsation Ostlie found

$$
\log \mathrm{P}_{\mathrm{O}}=-1.92-0.73 \log \mathrm{M}+1.86 \log \mathrm{R}
$$

and for first overtone pulsation

$$
\log P_{1}=-1.60-0.51 \log M+1.59 \log R \text {. }
$$


The location of the blue edge of the instability strip for Mira pulsation is not easily derived from the theoretical studies, as linear studies show both overtone and fundamental modes to be pulsationally unstable over a broad range of $\mathrm{L}$ and $\mathrm{T}_{\text {eff }}$. Taking the mode of pulsation of the Miras to be the fundamental mode, making the risky assumption that the mode with the greatest linear growth rate will be preferred, and assuming that the onset of Mira pulsation corresponds to the transition from overtone to fundamental mode, yields a theoretical Mira onset line, as is indicated in Figure 1. However, it is not clear that the linear growth rates contain the essential physics of mode selection, and there are not enough models to allow even this crude method to be used for the lower metallicity stars, so we treat the onset line as a mostly free parameter.

Figure 1. Representative evolutionary tracks from Equation 4, based on Iben's (1984) parametrization, together with mode switching loci based on linear growth rates from Ostlie (1982). Stars to the right of the "mode switch" line would be more likely to pulsate in the fundamental mode, to produce hydrogen emission lines, and hence to be classified as Mira variables.

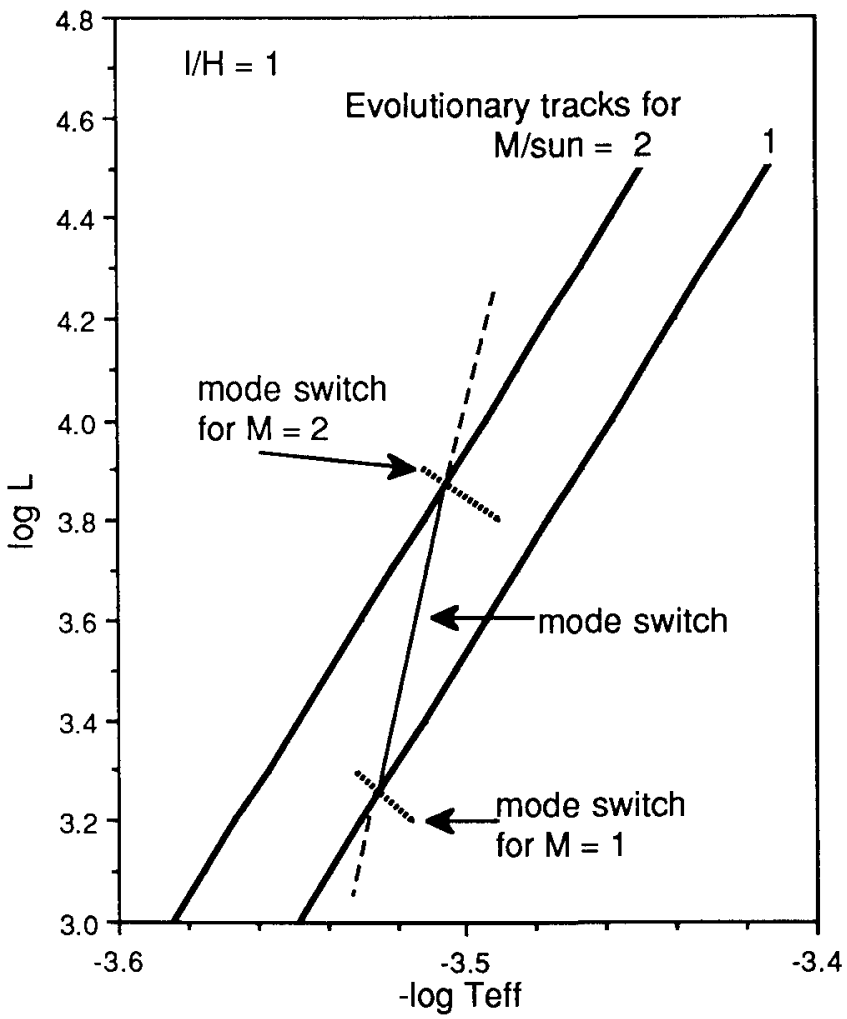


The "topography" of terminal AGB evolution. AGB stars follow a welldetermined luminosity-core mass relation during their H-shell burning "interpulse" phases, given roughly by $\mathrm{L}=6 \times 10^{4}\left(\mathrm{M}_{\mathrm{c}}-.52\right)$, and as a consequence evolve at a nearly constant rate in logL (e.g. Iben \& Renzini $1983=$ IR). This, coupled with the importance of mass loss during this phase, led Wood \& Cahn (1987) to suggest the use of a plot of $\log M$ vs. $\log L$ to show essential aspects of late AGB/Mira evolution. Such a plot is shown in Figure 2, again for the case $1 / \mathrm{H}=1$ and for solar metallicity. Included in this plot are lines of constant period (fundamental mode); the mode-switching line from Figure 1; and lines of constant $\mathrm{T}_{\text {eff }}$. Miras are expected to occupy some portion, possibly all, of the region between the $O \rightarrow F$ line and the $M=M_{\text {core }}$ line, with most of the Miras to be found where the evolution is slowest (i.e. where the mass loss rate is lowest, because without mass loss they all evolve to the right with roughly the same $d(\log L) / d t)$. From this diagram it is clear that we may expect to find Miras at a given period with a range of $M$ and $L$. Also, the relationship between the average $L$ or $M_{\text {progenitor }}$ and the period will depend on the distribution of Miras in this region particularly, on the mass loss function that affects the rate of evolution vertically in the diagram, and on the I(AGB)MF .

Figure 2. The "mode switch" line from Figure 1, lines of constant fundamental mode period $=200,300,400$ and 500 days, lines of constant effective temperature $=2950,3100 \mathrm{~K}$, and the core mass corresponding to each mass and luminosity have been computed using Equation 4 and $1 / \mathrm{H}=$ 1. Stars evolve to the right in this plot with $\mathrm{d}(\log \mathrm{L}) / \mathrm{dt} \sim 10^{-6} \mathrm{yr}^{-1}$ and downwards with $\mathrm{d}(\log \mathrm{M}) / \mathrm{dt}=\mathrm{M} / \mathrm{M}$.

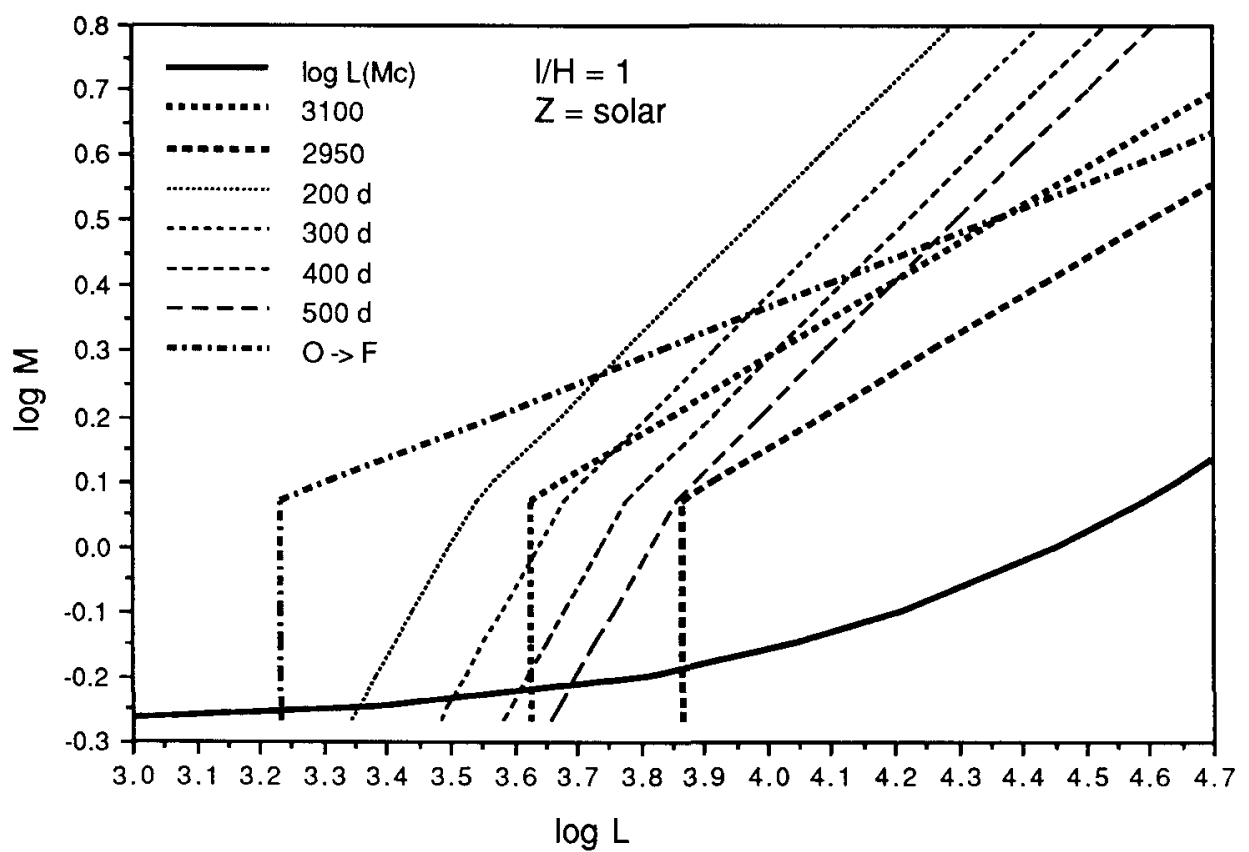


The $\log M$ vs. $\log L$ plot for a population with $Z=0.1 Z_{0}$ is shown in Figure 3; in this case there is no theoretical mode-switching line indicated, because there are not sufficient published models to use to derive one. It is clear that the effect of lowering $Z$ is to raise the luminosity at a given $M, P$. It is also clear that the typical effective temperatures will be higher for lower $\mathrm{Z}$ stars compared with higher $\mathrm{Z}$ stars with the same $P$, L or $M$, I.

The effect of a decrease in $1 / \mathrm{H}$ for either composition is to lower the effective temperature at a given $M, L$ or $M, P$, and to lower $L$ for a given $M, P$. Since the expected mass of a Mira at a given $P$ is less than the progenitor mass (constrained by observations) and the expectation that these stars are on their way towards $M=M_{\text {corer }} M$ is essentially fixed and either an increase in $1 / \mathrm{H}$ or a decrease in $\mathrm{Z}$ will raise the expected average $\langle\mathrm{L}\rangle(\mathrm{P})$ and $\left\langle\mathrm{T}_{\text {eff }}\right\rangle(\mathrm{P})$.

Since the dependence of the mass loss rate on stellar parameters for Miras is unknown, we approximate the effect on the evolution by assuming that the mass loss increases fairly abruptly when some critical combination of stellar parameters is reached, so that before this occurs, $d(\log M) / d t \ll d(\log L) / d t$ and after, $d(\log M) / d t \gg d(\log L) / d t$. This describes a "deathline" for the Miras; as they will spend relatively little time where $d(\log M) / d t$ is large.

Figure 3. As for Figure 2, but with $\mathrm{z}=0.1 \mathrm{z}_{0}$. Note that a given $(M, P)$ has higher $L$ for lower $Z$, and that Teff is also higher at a given $(\mathrm{L}, \mathrm{P})$ or $(\mathrm{M}, \mathrm{P})$.

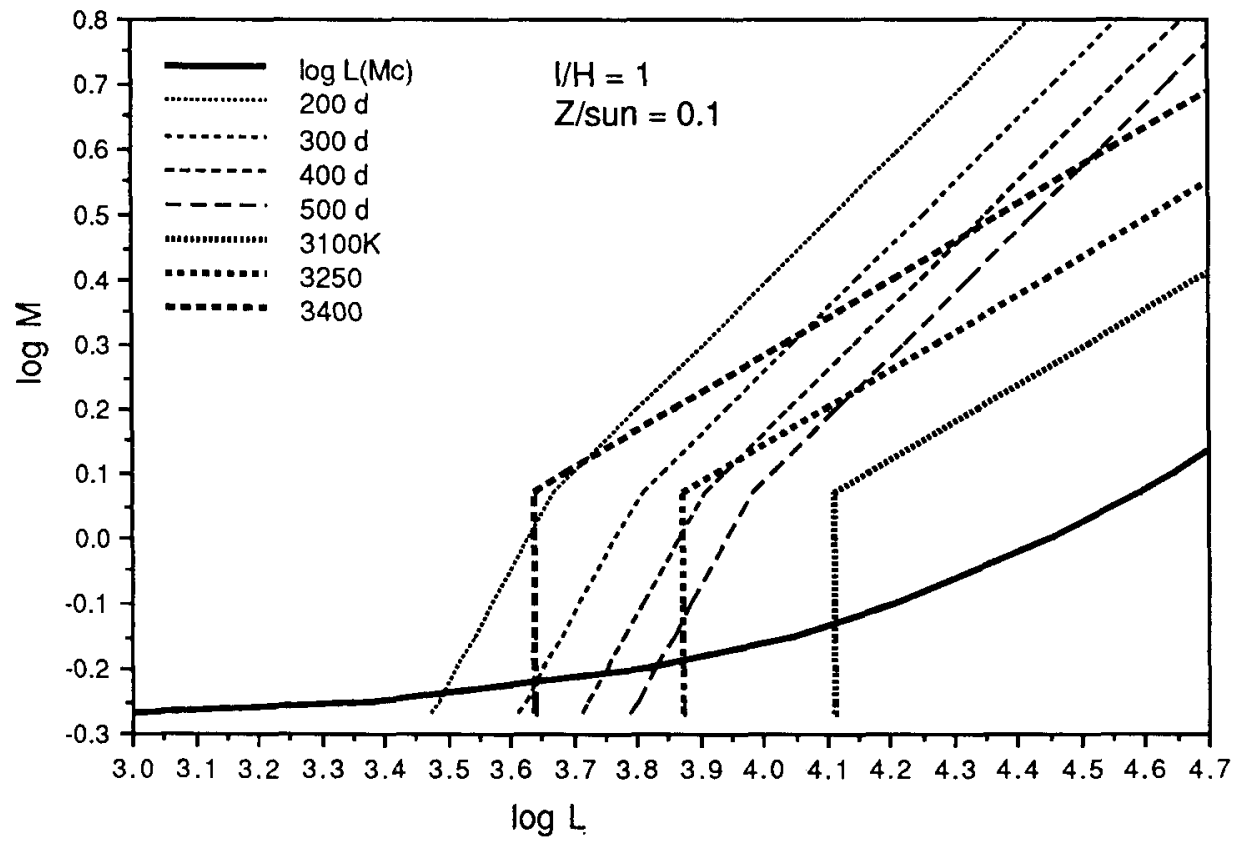


Thus most Miras will be found to the left/above the deathline, and the mass of the resultant white dwarf for a given star will be only slightly larger than $M$ at the deathline. This approach is motivated by several arguments favoring the existence of a relatively abrupt increase in the mass loss rate, termed the "superwind", at the end of the AGB.

The superwind phase. Studies of the relative number of white dwarfs in the solar neighborhood, of the occurrence of white dwarfs in relatively young clusters, and of the frequency of supernovae all indicate that stars with initial masses up to 5 to $8 \mathrm{M}_{\odot}$ eventually become white dwarfs with masses mostly less than $1 \mathrm{M}_{\ominus}$ and that the terminal white dwarf mass for a 3-5 $\mathrm{M}_{\odot}$ star is $0.7 \mathrm{M}_{\odot}$ (Weidemann 1984; see also IR). Comparisons of evolutionary models with observations indicate that most of this mass is lost while the stars are near the end of their AGB evolution. Models that incorporate steady, parametrized mass loss according to Reimers' relation cannot fit all the observational constraints with a single, constant, value for $\eta_{R}$ : if $\eta_{R}$ is too small, the calculations predict too many high luminosity $A G B$ stars and too many high mass white dwarfs, and if $n_{R}$ is too large, they predict too many low mass white dwarfs and not enough AGB stars. Iben (1984) pointed out that an average $\eta_{R}=1$ would prevent essentially all stars from reaching the $A G B$, and thus contradicts the fact that there are $S$ stars, $C$ stars and stars with atmospheric Technetium. In other words: any constant $\eta_{R}$ leads to too steep a dependence of the maximum $A G B$ luminosity on the initial mass of the star. The best agreement with observational constraints is obtained by assuming that the mass loss rate increases more precipitously than $L R / M$ near the end of the AGB. This enhanced mass loss "episode" has been termed the "superwind" by Renzini (1981a,b; see also IR).

The rapid increase in the mass loss rate that the observations suggest occurs near the end of the AGB evolution may in fact be necessary in order for a planetary nebula to be formed. Schonberner's (1983, 1987) evolutionary models indicate that a star will leave the AGB when its envelope mass is sufficiently small, with $M_{\text {ef }} \sim 0.01 M_{0}$ for a final core mass $\sim 0.55 \mathrm{M}_{\odot}$. The effective temperature of the post-AGB star depends mainly on its envelope mass. If only nuclear burning altered the envelope mass, then the star would take so long to evolve from $3000 \mathrm{~K}$ $\left(\mathrm{M}_{\text {env }} \sim 0.01 \mathrm{M}_{\odot}\right)$ to $5000 \mathrm{~K}\left(\mathrm{M}_{\text {env }} \sim 0.001 \mathrm{M}_{\odot}\right)$ that the mass ejected during the AGB stage would be long gone, and no planetary nebula would form. Schonberner $(1987,1989)$ invoked a continued "superwind" during the evolution from 3000 to $5000 \mathrm{~K}$, to reduce the envelope mass more rapidly. From our present understanding of the mass loss processes on the AGB, however, the mass loss rate should decrease abruptly as $\mathrm{T}_{\text {eff }}$ decreases, so this assumption is unrealistic. However, if the mass loss at the end of the AGB is sufficiently rapid, such that $t_{\dot{M}}=M_{\text {ef }} / \dot{M}<t_{K H}$ (where $M_{\text {ef }}$ is the final mass of the envelope), then the star will evolve blueward only at the rate given by $t_{K H}$; while the star is adjusting to accommodate the mass loss, the envelope mass may be reduced to an amount that corresponds to an effective temperature $>5000 \mathrm{~K}$. This may be easier for the more massive core remnants to achieve: Schonberner's models indicate that the difference between the envelope mass that induces blueward motion and that corresponding to $\mathrm{T}=5000 \mathrm{~K}$ decreases 
with increasing remnant masses.

The mechanism(s) of the superwind. Two factors determine $M$ in the dynamical models by Bowen: the effective temperature and the "driving amplitude" ( = the model parameter that governs the rate at which mechanical energy resulting from pulsation is being dissipated in the envelope). In the atmospheric models the effects of the internal driving are included through the imposition of an arbitrary inner "piston" boundary condition: at a position well below the photosphere but above the region of internal driving, the material is assumed to be moving with an imposed period, amplitude, and wave-form (usually sinusoidal). While the velocity structure of the lower atmosphere depends mostly on the period (or more precisely, on $Q=P \sqrt{\rho}$ ) and on the escape velocity, the density throughout the atmosphere also depends sensitively on the driving amplitude (Bowen 1988; WB). The mass loss rate is in turn very sensitive to the density distribution. For example, a Bowen model with $\mathrm{P}=400$ days, $\mathrm{M}=1.2 \mathrm{M}_{\theta}, \mathrm{T}=2925 \mathrm{~K}$ driven at $3 \mathrm{~km} \cdot \mathrm{sec}^{-1}$ had $\dot{M}=9 \times 10^{-8} \mathrm{M}_{\odot} \cdot \mathrm{yr}^{-1}$; the same model, driven at 4 $\mathrm{km} \cdot \mathrm{sec}^{-1}$, had $\dot{\mathrm{M}}=2.5 \times 10^{-6} \mathrm{M}_{\bullet} \cdot \mathrm{Yr}^{-1}$. The first mass loss rate is low enough to have little effect on the evolution; the second would dominate the evolution.

Generally, for a fixed piston amplitude, the mass loss rate increases exponentially near some temperature $T_{c}$ which is between 2900 and $3000 \mathrm{~K}$ for model and dust parameters that produce reasonably well-behaved atmospheric motions (for example, for piston amplitudes of $3-4 \mathrm{~km} \cdot \mathrm{sec}^{-1}$ in models similar to those described in Bowen 1988). The Mira models with $\mathrm{T}>\mathrm{T}_{\mathrm{c}}$ have small mass loss rates and thus evolve mainly in $\mathrm{L}$, while the ones with $T<T_{c}$ evolve mainly in $M$. This means that $T=T_{c}$ is a kind of "deathline" for Miras, where $\dot{M}$ passes $M_{*} / t_{e v}$. Physically, the existence of such a "critical temperature" is probably the consequence of the assumption that dust forms where the local radiative equilibrium temperature reaches some critical value; the ratio $R_{\text {dust }} / R_{\text {phot }}$ is determined almost entirely by $\mathrm{T}_{\text {eff }}$.

The critical temperature at which the mass loss rate becomes $\sim M_{\text {env }} / t_{\text {evol }}$ depends sensitively on the driving amplitude in the atmospheric models. Ostlie (1982) found that the linear growth rates became very large when $t_{\mathrm{KH}} / \mathrm{P}$ became small (< 10 to 100 depending on $\mathrm{M}_{*}$ ). While linear growth rates are not necessarily reliable indicators of the limiting non-linear behavior, ostlie did also find that non-linear models with small $t_{\mathrm{KH}} / \mathrm{P}$ were very unstable and tended to grow to very large amplitudes. Also, when the linear growth rates are large compared to the period, the star can probably oscillate in a more irregular way: the fundamental mode can increase and decrease in amplitude over only a few cycles, or there may be a more chaotic behavior. There are a number of stars whose light curves indicate that this is occurring (Cadmus et al. 1989). Such changes in the pulsation behavior are likely to enhance mass loss; at least, in the dynamical atmospheres, quick changes in the driving piston amplitude or waveform produce transients that tend to eject more material. Thus it is reasonable to consider the locus where the growth rates become large as a potential "deathline". The linear 
growth rates of the fundamental mode in the Ostlie models exceed $100 \% /$ cycle when the ratio $t_{K H} / P_{0}<1.815-1.400 \log M$. The line labeled "Lcrit" in Figures 4 and 5 shows where this value of $t_{K H} / P_{0}$ is reached.

Baud \& Habing (1983) proposed that the mass loss process be modeled with a mass loss rate that is inversely proportional to the envelope mass, so that the rate increases rapidly near the end of the AGB evolution. This was motivated by IRAS observations of AGB and post-AGB objects, that seemed to show a shortlived phase of high mass loss rates immediately preceding the end of the $A G B$ phase. They proposed that

$\dot{\mathrm{M}}_{\mathrm{BH}}=\dot{\mathrm{M}}_{\text {Reimers }} \mathrm{M}_{*} / \mathrm{M}_{\text {env }}$. The location where $\dot{\mathrm{M}}_{\mathrm{BH}}=\mathrm{M}_{\text {env }} / \mathrm{t}_{\mathrm{ev}}$ for the case with $\eta_{R}=$ is also marked in Figures 4 and 5 . While their relation is motivated by observations rather than by a physical mechanism, it clearly gives a similar "deathline" to the ones derived from the dust formation and the growth rate vs. $t_{\mathbf{K H}} / \mathrm{P}$ considerations.

Figure 4. Possible "deathlines" for solar composition AGB stars, computed using Equation $4 \mathrm{with} \mathbf{l} / \mathrm{H}=1$. Teff $=2950 \mathrm{~K}$ is a rough indicator of where the dust-driven mass loss becomes destructive in Bowen models with moderate driving. The line labeled " $\mathrm{L}_{\text {crit }}$ " is the locus where the linear growth rate of fundamental mode pulsation is expected to become large, based on the Ostlie (1982) models. The line labeled "BH" shows where $\dot{M}_{B H}=\eta_{R} 4 \times 10^{-13} \mathrm{LR} / \mathrm{M} \mathrm{M} \mathrm{M}_{\odot} \cdot \mathrm{Yr}^{-1}=\mathrm{M}_{\text {env }} / t_{\text {ev }}$ for the case $\eta_{R}=3 \cdot$. Each deathline maps (roughly) progenitor masses into final masses.

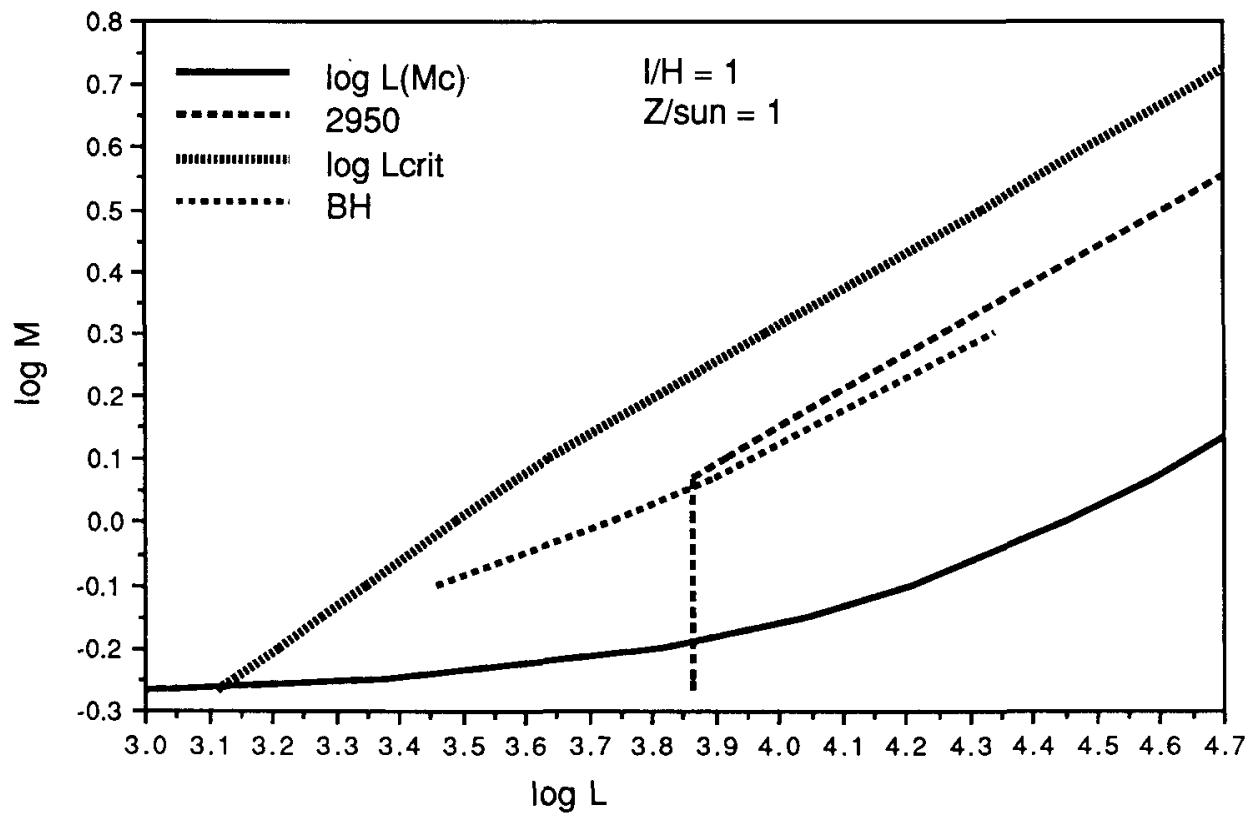


The three fundamental mode "deathlines" for Pop. I Miras are shown in Figure 4 for solar composition, and in Figure 5 for $z=0.1 \mathrm{Z}_{\odot}$, for $\mathrm{l} / \mathrm{H}$ $=1$ and assuming that the same values of $t_{K H} / P_{0}$ will be critical for both values of $\mathrm{Z}$. For Pop. I stars (and if $\mathbf{l} / \mathrm{H}>1$ ), the growth rate instability is probably the most important factor, because $\mathrm{T}_{\text {eff }}$ is still $>3000 \mathrm{~K}$ when stars reach this line. However, a slightly smaller $1 / \mathrm{H}$ results in a diagram where $\mathrm{T}_{\text {eff }}=2950$ occurs before the critical $t_{K H} / P_{0}$ is reached, so that the "dusty death" of a regular pulsator is still a viable possibility for Pop. I. For the lower Z Miras the "dusty death" line $T=2950 \mathrm{~K}$ has moved to higher $\mathrm{L}$ and longer $\mathrm{P}$, and thus the growth rate instability is probably even more important. I am not saying that the dust is unimportant in these cases; just that it is the growth in amplitude or irregularity of the oscillations that determines when the combined pulsation/dust mechanism becomes destructively efficient.

Note that Figure 5 indicates that there may be a class of supernovae in low metallicity populations that is less common or altogether absent in Pop. I: supernovae from stars of approximately $5 \mathrm{M}_{\odot}$ that reach the tip of the $A G B\left(\log L=4.7\right.$ or $\left.M_{\text {core }}=1.4\right)$ before they have a chance to

Figure 5. As for Figure 4 but for $z=0.1 \mathrm{Z}_{\odot}$. Also shown is the locus determined by the observed period-luminosity relation for the LMC, based on $\mathrm{m}_{\text {bol }}$ vs. $\mathrm{P}$ from Glass et al. (1987) with an assumed LMC distance modulus of 18.5 .

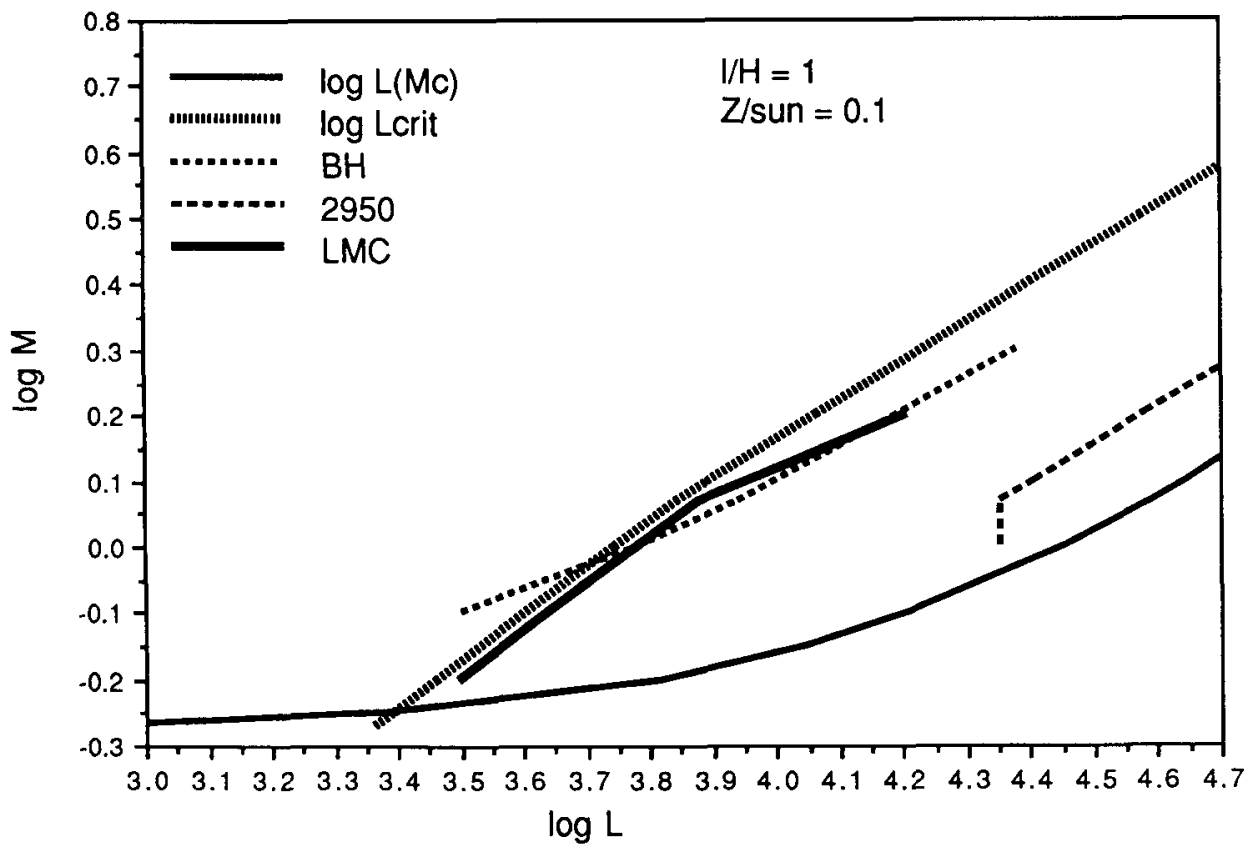


experience the superwind phase. This occurs for two reasons: (1) as can be seen in Figure 4, all the potential deathlines move to higher $\mathrm{L}$ at a given $M$ as the metallicity decreases; and (2) the ability of the atmospheric material to form sufficient dust to support the dusty wind will be less for lower metallicity stars (see also Jura 1986).

Are planetary nebulae "ejected"? It has been widely suggested that the $\overline{A G B}$ is terminated by the sudden "ejection" of a planetary nebula, either as the result of some sort of dynamical instability in the pulsating envelope, or as a consequence of a He shell flash. If by "ejection" is meant the removal of the envelope on a dynamical timescale, then the following facts argue that PNe are not "ejected":

First, if we assume that the excess mass of AGB stars is lost abruptly, after a gentle mass loss stage characterized for example by a Reimerstype relation with constant $\eta_{R^{\prime}}$ then choices of pre-Mira mass loss relations that account for the population and limiting luminosities of AGB stars lead to predictions of planetary nebula masses that are, on the average and in the extreme, higher than are observed. PN masses average $\sim 0.1 \mathrm{M}_{\odot}$ and extend to a maximum of $\sim 1 \mathrm{M}_{\odot}$, while the envelope mass needing to be removed exceeds 1-2 $\mathrm{M}_{\odot}$ in the stars with the more massive progenitors (e.g. Peimbert 1981). Of course one can get around this by carefully adjusting the pre-ejection mass loss function; but to get the right mass distribution in this case requires rather a lot of adjusting of the parametrized relations, with $\eta_{R}$ increasing rapidly with initial stellar mass, to leave just the right envelope mass to be "ejected" at the end.

Second, if this "ejection" is to occur simply as the result of instabilities in the envelope, there is only enough energy to eject the lower mass planetaries; to eject $1 \mathrm{M}_{\odot}$ of material in a dynamical time of $\leqslant 10$ years requires a mass loss rate of $0.1 \mathrm{M}_{\bullet} \cdot \mathrm{yr}^{-1}$; to accelerate this to a terminal velocity of $20 \mathrm{~km} \cdot \mathrm{sec}^{-1}$ would require $100 \%$ of the luminosity of the star (from Equation 1). While there is enough energy in a He shell flash to eject planetaries up to $1 M_{\theta}$, careful studies of the properties of $\mathrm{PNN}$ and $\mathrm{PNe}$ indicates that at most a few percent of these are formed while the star is burning He (Iben 1984; Schonberner 1989).

Third, observational studies of IRAS sources, OH-IR sources and proto-PNe, are providing constraints on the maximum mass loss rates of AGB stars, and these cluster around $10^{-4} \mathrm{M}_{\Theta} \cdot \mathrm{yr}^{-1}$ (Baud \& Habing 1983; Bedijn 1986, 1987). This is close to the maximum mass loss rate that can be driven by the combination of pulsation and radiative driving that accounts well for the mass loss from the Miras; it corresponds to the case with $\beta=\dot{M} / L_{C v} \sim 10^{1 \pm 1}$. Note that these maximum observed mass loss rates give characteristic mass loss time scales

$\left(M_{\text {env }} / \dot{M}\right) \sim t_{K H} \gg t_{\text {dyn }}$.

I rather prefer the idea that the mass loss rate increases continuously though perhaps precipitously during the stage of Mira/OH-IR pulsation, and that the observable planetary nebula is the result of the sweeping 
up of this outflow by the later, faster wind from the central stellar remnant, as originally explored by Kwok et al. (1978). This high rate of terminal AGB mass loss fits the requirements of Renzini's

"superwind". It also fits well with observations of some objects that have been tentatively identified as the immediate progenitors of planetary nebulae: the non-variable OH-IR sources as identified by Olnon et al. (1984). According to an analysis of IRAS observations by Bedijn (1986, 1987), these non-variable OH-IR sources typically consist of a relatively warm central star $(5-10,000 \mathrm{~K}$ or more) surrounded by a detached, outflowing remnant AGB wind. Characteristic ages for these, deduced from the flow speeds and the size of the cavity, are of the order of the Kelvin-Helmholtz time scale for the late AGB precursor, and deduced terminal mass loss rates of $\sim 10^{-4} \mathrm{M}_{\odot} \cdot \mathrm{Yr}^{-1}$ are consistent with $t_{M} \sim t_{K H} \sim 10$ to $100 t_{d y n}$ at the end of the AGB.

With steady mass loss, the mass of the planetary nebula is equal to the mass of the envelope at a time $\Delta t$ before the end of the AGB lequal to $\int \dot{M} d t$ over $\left.\Delta t\right)$. If the interval between the end of the AGB and the formation of the planetary nebula is sufficiently small, $\Delta t \approx R_{\text {neb }} / v_{\text {wind }}$. With $10^{-4} \mathrm{M}_{\odot} \cdot \mathrm{yr}^{-1}$ terminal $\mathrm{AGB}$ mass loss rate and $\mathrm{v}_{\text {wind }}=10 \mathrm{~km} \cdot \mathrm{sec}^{-1}$, the mass of the nebula when it reaches $R=0.1 \mathrm{pc}$ is $\leqq 1 \mathrm{M}_{\odot}-$ less if $M$ did not stay at $10^{-4} \mathrm{M}_{\odot} \mathrm{yr}^{-1}$ for the entire $\Delta t=10^{4}$ years or if there was a significant delay between the end of the wind and the beginning of the PN stage. A delay of 5000-7000 years is predicted from Schonberner's models if the mass loss process stops when $\mathrm{T}_{\text {eff }} \sim 5000 \mathrm{~K}$; this would result in a PN mass of just a few tenths of a solar mass. Thus the observed range of PN masses is quite consistent with the Kwok et al. colliding wind mechanism and the observed properties of terminal $A G B$ winds, combined with schonberner's post-AGB evolutionary models, as long as the star is able to stay cool, continue to pulsate and continue losing mass until $\mathrm{M}_{\text {env }} \sim 0.001 \mathrm{M}_{\odot}$.

\section{CONCLUSIONS}

The connection between stellar pulsation and mass loss for most classes of pulsating variable stars is in an early stage of investigation, and much work remains to be done to test the extent to which pulsation is implicated in important mass loss episodes for stars. Tantalizing results have been obtained from theoretical calculations for mass loss from Cepheid variables and from main sequence $A$ and $F$ stars; however, such mass loss has not yet been confirmed by observations.

For the Mira variables and the closely-related OH-IR stars, current modelling and recent observations (particularly from IRAS) have led to the identification of key mechanisms involved in stripping the envelopes from AGB stars to produce planetary nebulae and (mostly low mass) white dwarfs. The mass loss that produces planetary nebulae is seen to be a natural consequence of the pulsation combined with the production of dust in the stellar atmosphere. A prediction of this picture is that the mass loss process should allow a star of low metaliicity to reach a higher final AGB luminosity than a higher metallicity star with the same initial mass, and that as a result there may be a class of relatively 
low mass "AGB supernovae" that occur only in low metallicity populations.

Dynamical "ejection" is neither needed nor supported by observations; however the increasingly unstable pulsation behavior of models with decreasing $t_{K H} / P_{0}$ probably plays a key role in the production of the terminal "superwind". Planetary nebulae are proposed to be possible when the final AGB mass loss occurs sufficiently rapidly that $t_{\dot{M}}<t_{K_{H}}$ so that the star's envelope mass is brought below the minimum AGB envelope mass before the star has time to adjust its internal structure to have higher a $\mathrm{T}_{\text {eff }}$.

\section{BIBLIOGRAPHY}

Andreasen, G.K. (1988). Astron. \& Astrophys.,201, 72.

Aumann, H.H., Gillett, F.C., Beichman, C.A., de Jong, T., Houck, J.R., Low, F.J., Neugebauer, G., Walker, R.G. \& Wesselius, P.R. (1984). Ap.J., 278, L23.

Baud, B. \& H.J. Habing (1983). Astron. \& Astrophys.,127, 73.

Beach, T.E., Willson, L.A. \& Bowen G.H. (1988). Ap.J., 329, 241.

Becker, S.A. (1981). Ap.J.Suppl.,45; 33.

Bedijn, P.J. (1986). In Light on Dark Matter, ed. F. P. Israel, p. 119. Dordrecht: Reidel.

Bedijn, P.J. (1987). Astron. \& Astrophys.,186, 136.

Bessell, M.S. (1989). In Peculiar Red Giants, IAU Colloquium 106, ed. H.R. Johnson, in press.

Bowen, G.H., (1988). Ap.J., 329, 299.

Brugel, E.W., Beach, T.E., Willson, L.A. \& Bowen, G.H. (1987). In The Symbiotic Phenomenon, IAU, Colloquium 103, p. 67.

Brugel, E.W., Willson, L.A. \& Cadmus, R.R. (1986). In New Insights in Astrophysics, Proc. Joint NASA/ESA/SERC Conference, London, 14-16 July 1986, SP 263, p. 213.

Brunish, W.M. \& Willson, L.A. (1987). In Stellar Pulsation, Lecture Notes in Physics 174, eds. $\bar{A} . N$. Cox, W.M. Sparks \& S.G. Starrfield, p27. Berlin: Springer-Verlag.

Butler, C.J. (1987). In Circumstellar Matter, eds. I. Appenzeller \& C. Jordan, $2 \overline{27}$.

Cadmus, R.R. Jr., Willson, L.A. \& Sneden, C. (1989). In preparation.

Castor, J.I., Abbott, D.C. \& Klein, R.I. (1975). Ap.J.,195, 157.

Chiosi, C. \& Maeder, A. (1986). Ann. Rev. Astron. Astrophys.,24, 329.

Cox, A.N. (1980). Ann. Rev. Astron. Astrophys.,18, 15.

Fox, M.W. Wood, P.R. (1982). Ap.J.,259, 198.

Fox, M.W. \& Wood, P.R. (1985). Ap.J., 297, 455.

Gehrz, R.D. \& Woolf, N.J. (1970). Ap.J.,161, L213.

Gillett, F.C. (1986). In Light on Dark Matter, ed. F. P. Israel, p. 61. Dordrecht: Reidel

Glass, I.S., Catchpole, R.M., Feast, M.W., Whitelock, P.A., Reid, I.N. (1987). In Late Stages of Stellar Evolution, eds. S. Kwok \& S.R. Pot Tasch, p 51. Dordrecht: Reidel.

Hill, S.J. (1972). Ap.J.,178, 793. 
Hill, S.J. (1975). A.J.,80, 1044.

Hill, S.J. \& Willson, L. A. (1979). Ap.J.,229, 1029.

Iben, I. Jr. (1984). Ap.J.,277, 333.

Iben, I. Jr. \& Renzini, A. (1983). Ann. Rev. Astron. Astrophys., 21, 271. Jura, M. (1986). Ap.J., 301, 624 .

Knapp, G.R. (1986). Ap.J.,311, 731.

Knapp, G.R., Phillips, J.G., Leighton, R.B., Lo, K.Y., Wannier, P.G., Wooten, H.A. \& Huggins, P.J. (1982). Ap.J., 252, 616.

Kwok, S., Purton, G.R. \& Fitzgerald, P.M. (1978). Ap.J., 219, L125.

Little-Marenin, I.R. (1988). In Peculiar Red Giants, IAU Colloquium 106, ed. H.R. Johnson, in press.

McAlary, C.W. \& Welch, D.L. (1986). A.J.,91, 1209.

Olnon, F.A., Baud, B., Habing, H.J., de Jong, T., Harris, S. \& Pottasch, S.R. (1984). Ap.J.,278, L41.

Ostlie, D.A. \& Cox, A.N. (1986). Ap.J.,311, 864.

Ostlie, D.A. (1982). PhD Thesis, Iowa State University.

Peimbert, M. (1981). In Physical Processes in Red Giants, eds. I. Iben \& A. Renzini, p. 409. Reidel: Dordrecht.

Raveendran, A.V. \& Rao, K. (1988). Astron. \& Astrophys.,193, 259.

Reimers, D. (1975). In Problems in Stellar Atmospheres and Envelopes, eds. Basçhek, B., Kegel, W.H., Traving, G., p. 229. Berlin: Springer Verlag.

Reimers, D. (1987). In Circumstellar Matter, IAU Symposium 122, eds. I. Appenzelier \& C. Jordan, p. 307. Dordrecht: Reidel.

Renzini, A. (1981a). In Physical Processes in Red Giants, eds. I. Iben \& A. Renzini, p. 165. Dordrecht: Reidel.

Renzini, A. (1981b). In Effects of Mass Loss on Stellar Evolution, eds. C. Chiosi \& R. Stalio, p. 319. Dordrecht: Reidel.

Richer, H.B. (1989). In Peculiar Red Giants, IAU Colloquium 106, ed. H.R. Johnson, in press.

Rood, R.T. (1973). Ap.J., 184, 815.

Schmidt, E.G. \& Parsons, S.B.. (1984). Ap.J., 279, 202.

Schonberner, D. (1983). Ap.J., 272, 708.

Schonberner, D. (1987). In Late Stages of Stellar Evolution, eds. S. Kwol: \& S. R. Pottasch, p. 337.

Schonberner, D. (1989). In Peculiar Red Giants, IAU Colloquium 106, ed. H.R. Johnson. In press.

Smith, M.A. \& Penrod, G.D., (1985). In Relations Between Chromospheric/Coronal Heating and Mass Loss in Stars, eds. Stalio \& Zirker, p. 394. Italy: Tabographis.

Smith, M.A. (1988). In Pulsation and Mass Loss, eds. L.A. Willson \& R. Stalio, p. 251. Dordrecht: Reidel.

Stahler, S.W. (1983). Ap.J., 274, 822.

Stellingwerf, R.F. (1979). In Nonradial \& Nonlinear Stellar Pulsations, Lecture Notes on Physics 125, eds. H.A. Hill \& W.A.

Dziembowski, p. 50. Berlin: Springer Verlag.

Weidemann, V. (1984). Astron. \& Astrophys., 134, L1.

Welch, D.A. \& Duric, N. (1988). A.J., $95,17 \overline{94}$.

Willson, L.A. (1986). P.A.S.P.,98, 37.

Willson, L.A. (1988). In Pulsation and Mass Loss, eds. L. A. Willson \& R. Stalio, $\overline{\mathrm{p}}$. 285. Dordrecht: Reidel. 
Willson, I.A. \& Bowen, G.H. (1984). Nature, 312, 429.

Willson, L.A. \& Bowen, G.H. (1985). In Relations Between Chromospheric/Coronal Heating and Mass Loss in Stars, eds. R. Stalio \& J.B. Zirker, p. 127. Italy: Tabographis.

Willson, L.A. \& Bowen, G.H. (1986a). Irish Astronomical Journal, 17, 249. Willson, L.A. \& Bowen, G.H. (1986b). In Cool Stars, Stellar Systems, and the Sun, Lecture Notes in Physics Vol. 254, eds. M. Zeilik \& D. M. Gibson, p.385. Berlin: Springer-Verlag.

Willson, L.A. \& Hill, S.J. (1979). Ap.J.,228, 854.

Willson, L.A. \& Kowalsky, P. (1987). In Late Stages of Stellar Evolution, eds. S. Kwok \& S. R. Pottasch, p. 277. Dordrecht: Reidel.

Willson, L.A., Bowen, G.H. \& Struck-Marcell, C. (1987). Comments on Astrophysics, XII, pp 17.

Wood, P.R (1979). Ap.J., $227,220$.

Wood, P.R. \& Cahn, J.H. (1977). Ap.J.,211, 499.

Wood, P.R., Bessell, M.S. \& Fox, M.W. (1983). Ap.J.,272, 99. 